\title{
Laïcité et société post-séculière
}

\section{Roger Monjo}

\section{(2) OpenEdition}

Journals

Édition électronique

URL : http://journals.openedition.org/trema/2710

DOI : 10.4000/trema.2710

ISSN : 2107-0997

\section{Éditeur}

Faculté d'Éducation de l'université de Montpellier

\section{Édition imprimée}

Date de publication : 1 avril 2012

Pagination : $72-87$

ISSN : 1167-315X

\section{Référence électronique}

Roger Monjo, «Laïcité et société post-séculière », Tréma [En ligne], 37 | 2012, mis en ligne le 01 avril 2014, consulté le 19 avril 2019. URL : http://journals.openedition.org/trema/2710 ; DOI : 10.4000/ trema. 2710

Ce document a été généré automatiquement le 19 avril 2019

Trema 


\section{Laïcité et société post-séculière}

\section{Roger Monjo}

Le dialogue n'est véritable que si l'on éprouve la tentation d'épouser le point de vue

d'autrui.

Jean LACROIX

(Le sens du dialogue, La Baconnière, Neuchatel, 1944)

\section{Introduction}


1 Le préfixe « post » est désormais largement mobilisé pour caractériser les sociétés contemporaines: des sociétés postmodernes, post-industrielles ou postsalariales, post-nationales et postcoloniales, mais aussi post-séculières. La première de ces formulations est, manifestement, la plus générale dans la mesure où chacun des traits qui est successivement mis en exergue par les formulations suivantes peut être considéré comme désignant, en négatif, une

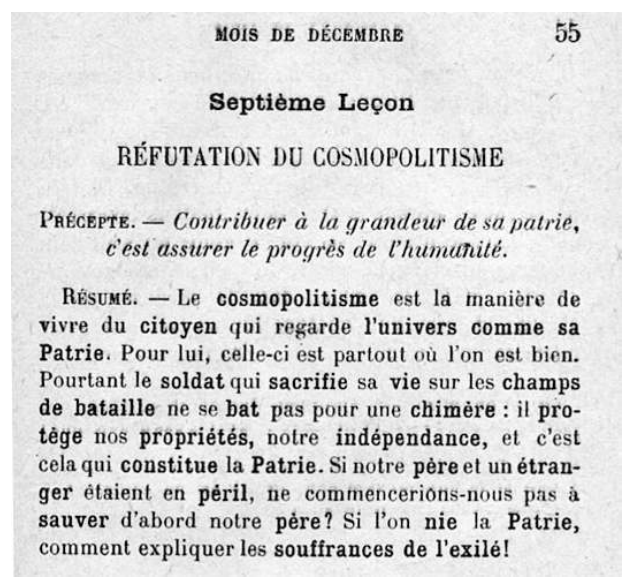
dimension majeure, ou du moins importante, des sociétés "modernes » : l'hégémonie du travail industriel et du régime salarial dans lequel il s'est déployé, l'apparition de l'État-nation et son expansionnisme, sous sa forme occidentale en particulier, la sécularisation (ou le « désenchantement du monde ») et, plus précisément, pour la France, la laïcisation du lien social et politique.

2 Autant de processus historiques importants qui ont marqué l'émergence et le développement des sociétés dites "modernes » en raison de leur inscription, plus ou moins délibérée, dans un projet: le projet de la modernité, précisément, tel qu'il est formulé et théorisé au XVIIIe siècle, par les philosophes des Lumières en particulier. Un projet d'émancipation d'abord, qui reposa sur la confiance faite à la raison humaine pour éradiquer toute forme d'aliénation ou de domination injustifiée, mais qui prit aussi la forme d'un procès de rationalisation du monde, producteur de multiples effets qui se sont, à la longue, révélés " pervers » : la généralisation de l'exploitation du travail humain (mais aussi de la nature) dans le cadre de ce salariat que MARX n'hésitait pas à assimiler à une nouvelle forme d'esclavage, l'ethnocentrisme occidental qui, sous sa forme nationaliste, a été à l'origine des affrontements les plus meurtriers que l'humanité ait connus, la levée progressive de tous les freins moraux et, éventuellement, politiques que la religion et, plus largement, le souci du sacré et de la spiritualité pouvaient opposer au déploiement sans limites de l'égoïsme individuel.

3 Aussi la question est-elle d'abord d'interprétation : faut-il entendre, dans ces différentes formes de dépassement de la modernité, l'expression d'une volonté plus ou moins délibérée de revenir sur ces débordements et, en jetant le bébé avec l'eau du bain en quelque sorte, de renoncer au grand récit de l'émancipation en raison de son lien inévitable avec les pires dérives que l'histoire humaine ait connues? Un retour, en somme, aux formes pré-modernes du lien social serait alors visé : décroissance, démondialisation dé-marchandisation, voire dé-monétarisation, de la sphère de la production et promotion de l'autarcie économique, réactivation des communautés «chaudes», locales et familiales, "ré-enchantement du monde», etc. Les "Temps Modernes " apparaîtraient alors, in fine, comme une sorte de parenthèse, éphémère, encadrée par des époques «pré-modernes » (mais aussi pré-industrielles, pré-politiques, etc.) et « post-modernes ", qui partageraient, au fond, une même sagesse construite sur le renoncement, par anticipation pour les premières ou instruit par l'expérience pour les secondes, aux ambitions prométhéennes («nous rendre maîtres et possesseurs de la nature ») de l'époque moderne mais aussi à leurs effets délétères sur la condition humaine. Ou bien faut-il comprendre ce «dépassement» dans un sens plus hégélien (comme 
Aufhebung) : un prolongement du processus de la modernité, mais qui serait repris sur un autre mode, plus réflexif et plus averti des dérives possibles, de telle sorte que le projet de l'émancipation ne serait pas abandonné mais, au contraire, conduit à son terme dans la mesure où les «effets pervers" sont alors mis sur le compte, en réalité, du caractère encore inabouti de ce projet? La modernité comme « projet inachevé » pour reprendre la formule de J. HABERMAS.

En rapprochant alors l'expression "post-moderne» d'autres appellations voisines (modernité tardive, seconde modernité, modernité réflexive...), l'hypothèse suivante pourrait être adoptée : dans un premier temps, la promotion des valeurs spécifiquement modernes (l'autonomie, la raison, l'égalité, etc.) s'est traduite par l'élaboration de dispositifs, d'institutions et de normes qui ont emprunté beaucoup de leurs traits au monde pré-moderne, traditionnel, qui l'a précédée. Ce n'est qu'ultérieurement (seconde modernité) que cette traduction elle-même est appelée à se moderniser (pour de bon), en s'arrachant définitivement de cette gangue archaïque qui l'avait accompagnée dans les premiers temps ${ }^{1}$. On le verra, c'est là le fond de la thèse de ceux qui, évoquant la « catholaïcité » (néologisme forgé par Jean вЕAUвÉROT), voient dans le surgissement historique de la laïcité à la française une réplique à une hégémonie cléricale et religieuse, pesante voire aliénante, à laquelle elle emprunte, cependant, l'essentiel de ses dispositions et de ses procédures. De telle sorte que les débats récurrents, aujourd'hui, au sujet de cette question seraient d'abord l'indice de la nécessité d'un pas supplémentaire à franchir dans le sens de la sécularisation définitive de notre société, mais un pas qui, selon un paradoxe qui n'est qu'apparent, impliquerait la reconnaissance de certaines réalités religieuses comme appartenant de droit à l'espace public.

Plus globalement, cette hypothèse commande toute l'argumentation développée par Marcel GAUCHET dans un article déjà ancien et dont le titre lui-même ("L'école à l'école d'elle-même») en résume l'esprit. On peut y lire, par exemple, les formulations suivantes :

«[...] l'école, découvre-t-on (...) a fonctionné durant plusieurs siècles, pour sa partie consciente, sur des modèles idéaux rigoureusement rétrogrades par rapport aux présupposés latents déterminant son existence. Elle s'est constituée et perpétuée en reconduisant un système de normes anciennes à l'intérieur de ses articulations de base les plus nouvelles. Ce qui s'est passé depuis une trentaine d'années a consisté sous ce point de vue dans une remise en cause de cette part passéiste de l'institution... Ce qui est demandé en fait à l'école, au plus profond, c'est de rejoindre dans le contenu et les méthodes de son action les raisons qui commandent son existence - c'est d'aligner son fonctionnement explicite sur son fondement implicite. » (GAUCHET, 1985b, p. 79)

6 L'agitation actuelle autour de la question de la laïcité scolaire, mais aussi au-delà de l'école, dans la vie quotidienne de nos concitoyens, ne serait donc qu'un symptôme des inévitables difficultés que rencontre la marche de l'histoire quand elle approche du moment où une nouvelle étape doit être franchie, dans le sens de l'émancipation collective.

7 En nous inscrivant délibérément dans la perspective de cette deuxième hypothèse ${ }^{2}$, nous nous emploierons, dans un premier temps, à dégager l'originalité de la notion de société post-séculière en posant, en particulier, la question de l'articulation de cette notion avec les théories traditionnelles de la sécularisation. Se réduit-elle, en raison de l'importance qu'elle accorde à la question de la présence de la religion dans l'espace public, au constat, souvent effrayé, d'un «retour du religieux » pensé sur le modèle du retour du refoulé ? 
Désigne-t-elle alors le risque d'une régression majeure par rapport au projet moderne d'une pacification des relations sociales obtenue grâce à la relativisation et à la privatisation des multiples appartenances religieuses et, plus généralement, culturelles dont les individus peuvent se réclamer? Ou bien s'agit-il, au contraire, sur le modèle d'une post-modernité pensée comme hyper-modernité (ou seconde modernité), d'un approfondissement du processus de sécularisation lui-même, mais un approfondissement qui impliquerait la levée de l'ostracisme laïciste dont les convictions religieuses avaient été initialement victimes?

Nous aborderons, dans un deuxième temps, la question de l'application du principe de laïcité dans un tel contexte post-séculier. Qu'en est-il de cette application dès lors que la laïcisation de la société, du moins dans le sens que ce projet a pris dans le cadre de l'idéologie française, n'apparaît plus comme le telos de la modernité? Mais aussi, l'engouement que semble susciter cette caractérisation des sociétés contemporaines comme post-séculières ne nourrit-il pas quelque dangereuse connivence avec les dérives communautariennes actuelles, surtout lorsqu'elles sont d'inspiration religieuse, en leur assurant, en quelque sorte, une légitimité intellectuelle? Nous pourrons ainsi nous interroger sur les remaniements que l'adoption de cette approche post-séculière ne manquerait pas d'induire dans le champ de l'éducation dans la mesure où la laïcité s'est imposée, dans la tradition républicaine en particulier, comme l'une de ses valeurs fondatrices. La perspective post-séculière emporte-t-elle, avec elle, la remise en cause radicale de cette tradition?

\section{Qu'est-ce qu'une société post-séculière?}

8 Le philosophe allemand J. HABERMAS sera ici notre principale référence car c'est lui qui a introduit cette notion de société post-séculière dans le débat philosophique et public ${ }^{3}$. Dans son article pour Le Débat, il commence par rappeler cette évidence qui ne serait qu'une lapalissade s'il n'y ajoutait une précision décisive : « Une société post-séculière doit avoir été séculière ». Mais en indiquant aussitôt qu'une société post-séculière est une société qui continue d'être orientée par le processus de la sécularisation, mais un processus parvenu à un stade suffisamment avancé pour que des perspectives inédites s'ouvrent dans l'espace public, qui concernent la place du religieux et des religions dans cet espace. Car, les trois hypothèses qui sous-tendaient la conception classique de la sécularisation (le progrès technique-scientifique favorise une compréhension anthropocentrique d'une réalité "désenchantée ", parce que sujette, en totalité, à une explication causale; l'emprise des Églises sur la société recule en raison de la privatisation de la croyance religieuse ; le progrès économique et social est porteur d'une promesse de bien-être qui rend moins prégnant le besoin de faire appel à un «au-delà » réconfortant) sont aujourd'hui remises en cause et la conviction séculariste de la disparition programmée de la religion est profondément ébranlée au regard de la vitalité croissante que connaissent certains mouvements religieux, en particulier ceux qui sont animés par une visée universaliste, dans différentes régions du monde.

9 Certes, le processus de la sécularisation est bien toujours à l'œuvre dans une société postséculière dans la mesure où cette dernière reste une société de la "sortie de la religion", pour reprendre une formule du philosophe Marcel GAUCHET (1985a). Mais, cette «sortie de la religion », qui est l'autre nom de la sécularisation, signifie d'abord l'autonomisation de la décision politique (l'État) par rapport à la norme religieuse, incarnée par l'Église, de 
telle sorte que ce mouvement se confond largement avec celui de la démocratisation entendue comme autodétermination de la société. Cette "séparation" ne recouvre pas, contrairement à une représentation tenace mais erronée, le partage entre vie privée et vie publique, sauf à penser que cette dernière (le politique) se réduit à la sphère étatique. L'État, en tant qu'instance de la décision politique, suppose, pour pouvoir être considéré comme démocratique, que l'élaboration de cette décision est le résultat d'un débat public auquel chaque citoyen aura pu participer librement, dans le respect de certaines règles qui régissent la forme mais non le fond des arguments qui peuvent être avancés dans le cadre de la discussion publique. De telle sorte que, si les convictions religieuses ne peuvent plus, en tant que telles, s'avérer déterminantes pour les choix politiques d'une communauté qui se veut démocratique, elles n'en continuent pas moins d'être recevables dans la discussion collective qui va préparer l'élaboration de ces choix, dès lors qu'elles parviennent, à terme, à s'exprimer dans un langage profane. Pour le dire autrement : la sécularisation signifie moins l'éradication de la religion que l'atténuation croissante de son influence directe sur les orientations et les choix politiques de la société. Mais son influence reste possible, voire souhaitable, si elle se fait indirecte ou médiate. Dès lors que les convictions religieuses qui motivent certaines interventions ou prises de position dans le débat politique parviennent à s'exprimer à travers une argumentation publiquement recevable, c'est-à-dire compréhensible par tous, elles ont droit de cité au même titre que les convictions agnostiques ou athées.

Il est vrai que, dans un premier temps, cette volonté de limiter l'influence de la religion (et de l'Église) a pris un tour conflictuel, agonistique. Particulièrement en France où, pour des raisons historiques, le processus de la sécularisation a donné naissance au laïcisme, cette apologie de la laïcité qui s'est présentée sous la forme d'une contre-religion mais qui, par là même, s'est constituée comme une religion alternative (les Écoles normales, ces sanctuaires de la formation des maitres de l'école de la République, pensées comme des séminaires laïques, l'instituteur comme le rival mimétique du curé...). Mais, dès lors que les instances religieuses ont signifié leur allégeance aux règles de l'État de droit démocratique (séparation des Églises et de l'État et neutralité religieuse de ce dernier, reconnaissance de certains droits individuels fondamentaux comme la liberté de pensée, usage public de la raison comme fondement de la normativité sociale, politique et juridique), leur participation au débat public a pu être reconnue comme légitime. La pluralisation religieuse des sociétés contemporaines a été (aurait dû être), elle-même, un facteur non négligeable de cette pacification ${ }^{4}$.

11 Une société post-séculière se caractérise donc par une double exigence. Elle exige des religions une sorte de modernisation qui passe par la mise en perspective de ce qu'elle considère comme leur vérité, et par l'adoption d'un regard autocritique sur leur propre histoire. C'est là une condition de leur participation à un espace public démocratique. Car :

«L'État constitutionnel doit non seulement agir de manière neutre par rapport aux visions du monde, mais il doit également reposer sur des fondements normatifs qui peuvent être justifiés au moyen de raisons elles aussi neutres par rapport aux visions du monde - c'est-à-dire postmétaphysiques » (HABERMAS, 2008a, p. 148).

Mais, en même temps,

«il ne peut pas exiger de ses citoyens religieux ce qui serait inconciliable avec une existence authentiquement vécue "dans la foi“" (ibid., p. 149). 

seulement par souci pour la tranquillité sociale mais aussi pour une raison «normative » : il doit protéger la liberté de foi et de conscience de chacun. De telle sorte qu'une société post-séculière exige, en retour, que la raison laïque, séculière, s'ouvre à la raison religieuse en renonçant au préjugé naturaliste et scientiste pour qui

« les convictions religieuses sont per se non vraies, illusoires ou dénuées de sens » (

ibid., p. 56-57).

Car, dans cette dernière perspective qui est celle de l'opposition typiquement moderne entre raison et religion, les formes de la croyance religieuse sont perçues comme des archaïsmes et la liberté religieuse y est moins comprise comme un droit fondamental que comme une

«mesure de protection culturelle en faveur d'espèces en voie de disparition » (ibid., p. 199).

Pour Jürgen Habermas, la raison séculière, tout en restant agnostique, peut se mettre à l'écoute, sans se renier, des convictions religieuses et leur reconnaitre une portée cognitive et morale, universelle, pertinente du point de vue des questions que se posent nos contemporains. En ce sens,

« la sécularisation a moins la fonction d'un filtre qui empêcherait le passage des contenus traditionnels que celle d'un transformateur qui modifie le courant provenant de la tradition » (ibid., p. 145) 5 .

La raison moderne est déjà parvenue, au demeurant, à traduire en langage laïc, compréhensible par tous, certaines des composantes morales de la tradition religieuse (par exemple, la re-formulation de la charité chrétienne en termes de solidarité sociale). On a pu aussi voir dans le marxisme et l'utopie communiste une reprise, sur de tout autres bases, du message évangélique. Et certains auteurs se réclamant, au XXe siècle, de cette utopie ont pu remettre en circulation, en les laïcisant, des thèmes empruntés à la tradition religieuse : par exemple, W. BENJAMIN reformulant la perspective du « jugement dernier » dans le projet d'une histoire écrite « du point de vue des vaincus » ${ }^{6}$ ou E. BLocH renouant avec le " principe espérance ». être traduits dans un discours au sein duquel l'effet d'arrêt qui s'attache aux vérités révélées peut être levé » (ibid., p. 158).

ais, plus encore aujourd'hui - c'est là un autre aspect important de l'argumentation de Jürgen HABERMAS - les religions, en particulier les religions du Livre et leurs longues traditions herméneutiques, peuvent se révéler être de précieuses ressources de sens dans un monde qui, comme le nôtre, est affecté de profondes pathologies sociales. Car l'idéologie dominante (mixte de scientisme, d'économisme et de technicisme), loin d'offrir des points d'appui pour combattre ces pathologies, voire, même simplement, des éléments de consolation pour les rendre supportables, les redouble plutôt en renvoyant chacun et d'abord les plus faibles et les plus démunis à un face-à-face solitaire avec elles. La science en particulier, cette idéologie des temps modernes, a ouvert des perspectives techniques (maitrise de l'énergie, biotechnologies...) qui posent des problèmes éthiques inédits (rapport aux ressources naturelles, fabrication du vivant, etc.) auxquels elle est bien incapable de répondre. Et, dans un monde marqué, par ailleurs, par l'épuisement de tout mouvement révolutionnaire, messianique ou utopique, fondé sur une philosophie de 
l'histoire (et du progrès) largement partagée, il est, en quelque sorte, naturel que la religion apparaisse comme une option recevable, donatrice de sens, pour beaucoup et d'abord pour tous les damnés de la terre. Car :

«[...] la raison pratique manque sa propre destination si elle n'a plus la force de faire prendre conscience aux cœurs profanes de ce que la solidarité est partout dans le monde offensée, si elle n'a plus la force d'éveiller et d'entretenir une conscience de ce qui manque, de ce qui scandalise» (ibid., p. 146).

Aussi, poursuit Jürgen HABERMAS, tout penseur «progressiste» qui se réclame de la philosophie des Lumières et de la raison et qui est par ailleurs soucieux d'une revitalisation de la vie démocratique aujourd'hui anémiée et motivé par le projet de « conjurer les menaces qui pèsent aujourd'hui sur le contenu normatif de la modernité » ( ibid., p. 47), c'est-à-dire le projet d'émancipation individuelle et collective, loin de mépriser les convictions religieuses au prétexte de leur archaïsme et de chercher à leur interdire tout accès à l'espace public au prétexte de leur dérive fondamentaliste, devrait, tout au contraire, encourager et leur faciliter cet accès, tout en les aidant à reformuler leur "potentiel critique" 7 dans le langage de la raison, un langage profane compréhensible et recevable par tous.

\section{Laïcité et société post-séculière}

Qu'en est-il alors, dans un tel contexte post-séculier, de l'application du principe de laïcité comme principe juridique?

Dans sa réponse, Philippe FORAY insiste tout particulièrement sur un point : la laïcité ne se réduit pas à la séparation de l'Église et de l'État (et à la loi de 1905). Elle implique aussi l'élaboration de (et l'adhésion à) un projet, politique, de vivre ensemble dans un « monde commun ", c'est-à-dire en partageant une culture commune. C'est ce qui justifie qu'on la regarde comme un principe constitutionnel (ou " principe de justice ", au sens de J. RAWLS ) dans la mesure où elle a pour fonction de garantir la coexistence des libertés individuelles, en particulier la liberté de conscience. Mais cette notion de «monde commun » est, à bien des égards, reconnaît Philippe FORAY, complexe et problématique. Son contenu mérite d'être précisé et c'est à cette fin qu'il s'emploie à explorer une voie intermédiaire, nécessairement étroite, entre une lecture strictement juridique (libérale, ou encore " négative ») du principe de laïcité qui s'en tient à la séparation de l'État et de l'Église, c'est-à-dire à l'exigence de neutralité religieuse de l'instance politique, et une lecture éthique-politique (celle du néo-républicanisme actuel, qui prétend renouer avec le laïcisme des origines) qui fait de la laïcité une véritable doctrine philosophique substantielle (une théorie du bien ou une "doctrine compréhensive ", pour reprendre à nouveau le vocabulaire de J. RAWLS) concurrente, dans sa dimension englobante, de toute doctrine religieuse. Si cette seconde approche va manifestement trop loin dans le sens de la moralisation de la laïcité et court ainsi le risque de relancer à nouveau la "guerre des deux France » en se donnant comme une véritable alternative à la (aux) religion(s), elle a malgré tout le mérite d'attirer l'attention sur un point important : la laïcité, en particulier dans sa dimension scolaire, ne se réduit pas à la seule neutralité vis-à-vis des confessions religieuses mais, je cite P. FORAY, « implique aussi une action positive que l'on peut décrire comme le projet de développer la liberté de penser de chacun ». De telle sorte que la voie intermédiaire entre neutralité (laïcité négative) et laïcisme (laïcité positive) qui est ici envisagée reposerait sur un équilibre, nécessairement fragile, entre liberté de conscience 
(liberté de croire ou de ne pas croire) et liberté de penser (formation au " penser par soimême » et à l'esprit critique revendiqués par la philosophie kantienne et, plus largement, la philosophie des Lumières qui a inspiré le projet de la modernités $)$.

21 Ainsi, la culture politique commune qui constitue l'autre versant, plus positif que le versant strictement juridique, de la laïcité reposera sur cette double exigence « libérale » : la reconnaissance du pluralisme, insurmontable aujourd'hui, des conceptions du bien d'une part, une reconnaissance qui rend possible la coexistence et la coopération sociale entre croyants (eux-mêmes pluriels) et non-croyants, et la défense de l'idée d'autonomie individuelle d'autre part, constitutive de la modernité et qui autorise chacun à se déprendre, à tout moment, de toute appartenance (religieuse, culturelle, philosophique, etc.) à laquelle il avait, jusque-là, marqué son adhésion.

C'est donc d'une «laïcité de confrontation " (ou "laïcité délibérative ») que se réclame Philippe FORAY, en se prononçant en faveur d'une reconnaissance de la légitimité des interventions religieuses dans le débat public. Il précise même, en référence aux analyses de Marcel GAUCHET, que cette légitimité est d'autant plus assurée aujourd'hui que la sécularisation continue de progresser: c'est précisément parce que nous vivons une période de «sortie de la religion » que les Églises redeviennent socialement visibles sans que ce retour constitue une menace pour la laïcité de l'État.

23 Mais c'est ici, précisément, que la difficulté surgit. À quelle forme de légitimité ces interventions religieuses dans le débat public peuvent-elles prétendre dès lors que le souci de l'autonomie individuelle, ce versant " positif» qui vient compléter la définition strictement juridique de la laïcité, est jugé lui-même comme partie prenante d'une vision du monde (une "théorie du bien» ou "doctrine compréhensive») avec laquelle une conviction religieuse peut se déclarer incompatible dans la mesure où elle fera, pour sa part, de l'appartenance à la communauté des croyants la valeur suprême, en concluant, du même coup, à une relativisation de cette même "autonomie"? Pour le dire autrement : comment organiser le vivre-ensemble entre croyants et non-croyants en leur faisant partager une même culture politique, d'essence libérale, si les premiers accordent à leur foi un rôle structurant pour l'ensemble de leur existence, y compris dans ses dimensions politiques, en récusant ainsi ce qui fait l'essence de la philosophie libérale? Ou encore et au risque de la caricature : jusqu'à quel point un État démocratique est-il en droit d'obliger certains croyants à mener une sorte de vie schizophrénique en les obligeant à séparer ce qui pour eux est inséparable, à savoir leur vie privée et leur vie publique ? La conviction selon laquelle la religion est une affaire strictement privée qui doit être maintenue dans les limites étroites d'une conscience individuelle maîtresse de ses propres croyances et orientations spirituelles est elle-même le résultat d'un long apprentissage historique qui n'a affecté que certaines sociétés, en particulier celles qui ont été confrontées aux drames engendrés par les "guerres de religion ». De telle sorte qu'une telle conviction repose toujours, plus ou moins explicitement, sur le présupposé selon lequel les croyances religieuses sont facteurs de discordes et que leur accorder une trop grande «publicité » mettra nécessairement en danger le lien de coopération sociale qui doit unir les membres d'une même communauté politique. Mais que faire face à des convictions religieuses qui ne se satisfont pas d'être seulement "tolérées", comme on tolère certaines déviances, certains écarts à la norme (ici, l'autonomie individuelle), dès lors qu'ils restent discrets, voire invisibles et ne remettent pas en cause le consensus normatif qui régit la vie en commun? Car : 
«Pour que le principe de tolérance soit affranchi du soupçon de fixer les limites de la tolérance dans une optique répressive, la définition de ce qui peut encore être toléré et de ce qui ne le peut plus exige des justifications ayant pour toutes les parties la clarté de l'évidence et pouvant être acceptées par elles " (HABERMAS, 2008a, p. 178). croyants car des «raisons fonctionnelles interdisent également qu'on réduise trop rapidement la complexité polyphonique dans le concert public » :

«(L'État libéral) ne peut pas décourager les croyants et les communautés religieuses de s'exprimer aussi politiquement en tant que tels, parce qu'il ne peut pas savoir si en procédant de la sorte il ne coupe pas la société séculière de ressources importantes pour la fondation du sens. Les citoyens laïques ou ceux qui appartiennent à d'autres religions peuvent toujours, dans certaines circonstances, apprendre quelque chose de contributions religieuses, ne serait-ce qu'en reconnaissant, par exemple, dans les contenus de vérité normatifs d'un énoncé religieux certaines de leurs propres intuitions parfois enfouies » (ibid., p. 190).

ce « devoir de civilité » s'impose aussi (surtout?) aux laïques pour des raisons qui leur sont propres, car elle sont liées à l'histoire de la raison séculière elle-même, et qui vont au-delà du nécessaire " respect » dû aux convictions qui nous sont étrangères. Ces raisons se dévoilent dans la recommandation suivante :

«[...] la modernité doit surmonter par l'autoréflexion la compréhension limitée que lui donne d'elle-même un laïcisme endurci et exclusif » (ibid., p. 199). conceptions religieuses ne pourront que disparaître, tôt ou tard et en proportion des 
progrès que la connaissance scientifique continuera de réaliser. Ils doivent apprendre à reconnaître que leur non-adhésion aux conceptions religieuses est à l'origine d'un dissensus « qu'il est raisonnable d'escompter ». Et, dans la mesure où c'est d'abord leur «foi » en la science qui semble être à l'origine de ce «laïcisme endurci et exclusif», cet apprentissage sera guidé par la question suivante :

«la science moderne est-elle une pratique qui ne se comprend entièrement qu'à partir d'elle-même et établit performativement le critère de tout ce qui est vrai ou faux, ou bien faut-il plutôt la comprendre comme le résultat d'une histoire de la raison qui inclut tout autant les religions universelles ?» (ibid., p. 210).

31 Un double apprentissage donc, qui ne permettra probablement pas un rapprochement des points de vue, encore moins l'élaboration d'un consensus, car :

«[...] les conflits qui menacent d'éclater ne portent plus sur des biens matériels faisant l'objet d'un consensus, mais sur des biens spirituels en concurrence les uns avec les autres. Les conflits existentiels axiologiques entre communautés de foi ne sont pas susceptibles de compromis » (ibid., p. 195).

Mais un double apprentissage qui aura des vertus civilisatrices dans la mesure où il fera du dialogue et de la discussion à la fois le but et le moyen. De ce point de vue, la réflexion de HABERMAS ne se limite pas à la perspective strictement procédurale à laquelle on la réduit trop souvent et il est lui-même tout à fait conscient des limites de cette perspective :

«[...] il ne va pas de soi du point de vue des motivations qu'une entité politique réunissant une pluralité de visions du monde parvienne à se stabiliser normativement sur la simple présomption que, au-delà d'un simple modus vivendi, puisse se réaliser en arrière-plan une entente ne portant que sur des procédures et des principes, et donc une entente simplement formelle » (ibid., p. 153).

33 C'est pourquoi, si je partage la «réticence» exprimée par P. FORAY vers la fin de sa réponse, à l'égard du «formalisme qui (lui) semble impliqué par un déplacement conduisant d'une polarisation sur l'objet de la discussion et la recherche d'un accord sur cet objet à une polarisation sur la pratique de la discussion elle-même ", je dois ajouter que ce n'est précisément pas la perspective dans laquelle je m'inscris, ni celle qui est privilégiée par Jürgen HABERMAS lui-même. Et, s'il est vrai que la discussion devient alors, elle-même, un « Bien », au-delà des « Biens » qui en sont l'objet, c'est au sens où, loin de constituer la diversion qui permettrait d'occulter ce que la confrontation de points de vue définitivement inconciliables peut avoir de "tragique», elle permet au contraire d'apprendre à vivre (ensemble) avec ce tragique dans la mesure où elle devient par excellence le lieu où s'opère cette reconnaissance (réciproque) de l'autre, dans sa différence. C'est bien parce qu'elle est le lieu d'un tel apprentissage (simultané) qu'elle peut être au centre d'un projet éthique.

C'est pourquoi il n'y a pas, à mon sens, de véritable rupture entre les perspectives de Jürgen HABERMAS (du moins dans ses textes les plus récents) et celle d'Axel HONNETH. S'il est vrai, comme le souligne Philippe FORAY en référence à ce dernier, qu'il convient d'accorder une attention toute particulière à "l'expérience du mépris ", dès lors que, compris comme "déni de reconnaissance ", il est à la source d'un sentiment d'injustice que l'offre de réponses matérielles ne suffira pas à soulager, il me semble que l'exclusion de la discussion publique, au prétexte de convictions religieuses trop "ostentatoires ", constitue la forme première et paradigmatique de cette expérience ${ }^{9}$. Car :

«Le processus démocratique doit sa force de conviction légitimante au fait qu'il intègre toutes les personnes concernées, mais il le doit surtout à son caractère 
délibératif, car c'est sur cette base que l'on peut à juste titre supposer l'obtention de résultats rationnels à longue échéance » (HABERMAS, 2008a, p. 193).

\section{Conclusion}

La voie est étroite, en effet, et semée de difficultés, entre le renoncement pur et simple à tout projet politique d'émancipation fondé sur la raison et l'autonomie individuelle et la volonté de réactiver à tout prix le projet républicain dans sa forme initiale, laïciste, dans laquelle toute forme de croyance religieuse est synonyme d'aliénation et doit donc être éradiquée au profit d'un athéisme triomphant. D'autant plus étroite même qu'elle ne se décrètera pas et qu'aucune décision politique ne saurait la rendre obligatoire :

«[...] la théorie politique doit donc laisser ouverte la question de savoir si les mentalités fonctionnellement nécessaires peuvent être acquises par la voie de processus d'apprentissage [...] Pour que la réponse libérale au pluralisme religieux puisse être acceptée comme la réponse juste par les citoyens eux-mêmes, il faut [...] que les citoyens laïques et religieux acceptent d'aller, chacun de son point de vue, vers une interprétation du rapport entre foi et savoir, c'est la seule démarche qui puisse leur offrir de se rapporter les uns aux autres dans la sphère publique politique dans une relation éclairée par l'autoréflexion » (ibid., p. 208-211).

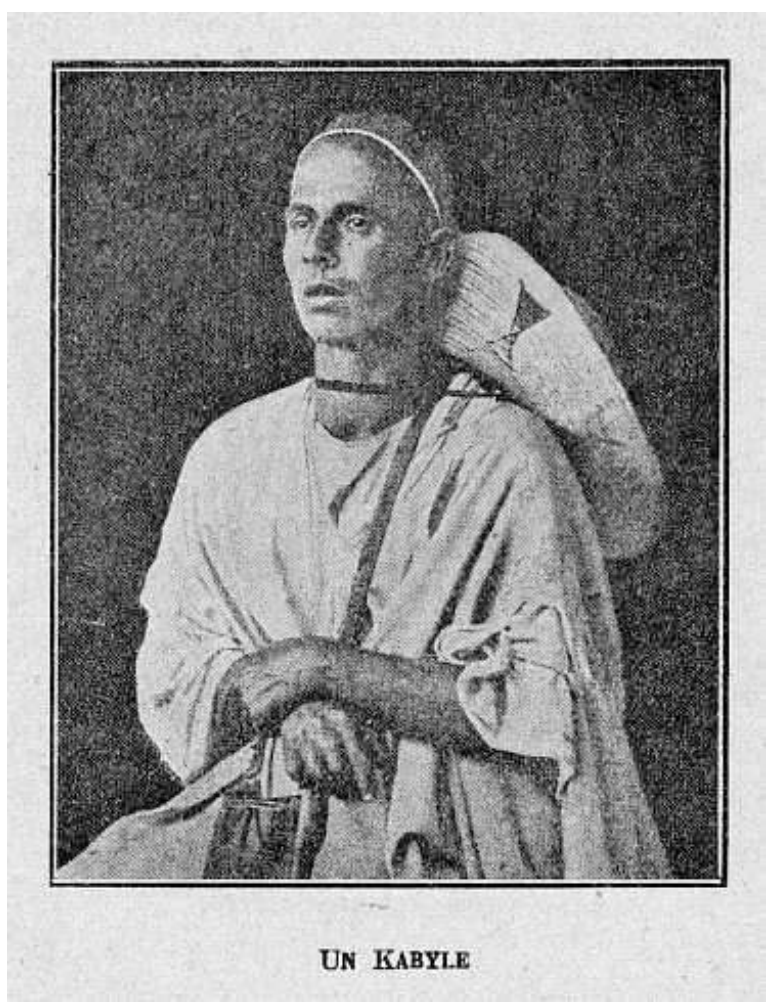

L'Algérie : Histoire, colonisation, géographie, administration, à l'usage des écoles primaires, P. BERNARD, F. REDON (1923). Source : CEDRHE. « un Kabyle», p. 161. 


\section{Chapitre $\mathrm{X}$ \\ LES INDIGENNES \\ RÉSUMÉ}

1. - La population indigène se multiplie rapidement depuis la conquête. Les Indigènes étaient 2.200 .000 en 1870 , ils sont aujourd'hui 5 millions.

2. - Les races indigènes sont au nombre de deux : la race berbère et la race arabe.

3. - Les Berbèreśs (Kabyles, Chaouĩas, Mzabites, Touaregs) sont les plus anciens indigènes de l'Afrique du Nord. Leur langue ne s'est conservée que dans le Djurjura, l'Aur'ès et le désert.

4. - Les Berbères sont en général sédentaires et agriculteurs.

5. - Les Arabes sont les descendants des conquérants du $7^{\circ}$ et du $11^{\mathrm{e}}$ siècles. Ils habitent les Hauts' Plateaux et les plaines du Tell.

6. - Les autres Indigènes de l'Algérie sont : les Maures, les Coulouglis, les Nègres, etc.

7. - Tous les Indigènes de l'Algérie sont Musulmans.

L'Algérie : Histoire, colonisation, géographie, administration, à l'usage des écoles primaires, P. BERNARD, F. REDON (1923). Source : CEDRHE. «Chapite X: Les indigènes », p. 159.

\section{BIBLIOGRAPHIE}

FERRY (Jean-Marc), « Sur le potentiel critique des religions dans l'espace européen », in GISEL P. et TETAZ J.-M. (Ed.), Théories de la religion. Genève : LABOR et FIDES, 2002.

FERRY (Jean-Marc), La religion réflexive, Paris, CERF, 2010.

FORAY (Philippe), La Laïcité scolaire, Berne, Peter LANG, 2008.

FORAY (Philippe), «Laïcité et bien commun. Réponse à Roger MONJO ", in Penser l'éducation, 끄26, Université de Rouen, décembre 2009.

GAUCHET (Marcel), Le désenchantement du monde, Paris, GALLIMARD, 1985a.

GAUChEt (Marcel), « L'école à l'école d'elle-même », in Le Débat, no 37, Paris, GALLimARD, 1985b.

HABERMAS (Jürgen), « Foi et savoir » in L'avenir de la nature humaine, Paris, GALLIMARD, 2002.

HABERMAS (Jürgen), « De la tolérance religieuse aux droits culturels », in Cités, no 13, Paris, PUF, 2003.

HABERMAS (Jürgen), « Religion et sphère publique », in Entre naturalisme et religion, Paris, GALLIMARD ,2008a. 
HABERMAS (Jürgen), « Qu'est-ce qu'une société post-séculière » ?, in Le Débat, no 152, Paris, GALLIMARD, 2008b.

MONJO (Roger), « École et foulard. La laïcité est-elle une doctrine politique moderne ? », in Penser l'éducation, nº 25, Université de Rouen, mai 2009.

\section{NOTES}

1. Juste un exemple : le mariage civil, lorsqu'il a « remplacé » le mariage religieux et même s'il a rompu avec l'indissolubilité de ce dernier, a reconduit, sur un autre plan, la ritualisation de l'engagement conjugal. C'est le PACS, aujourd'hui, qui incarne, sur un mode " hypermoderne », la nature strictement contractuelle de ce dernier.

2. Cette contribution s'inscrit dans le prolongement d'une discussion que j'ai eu le plaisir d'entretenir avec Philippe FORAY, Professeur à l'Université Jean MONNET de Saint-Étienne, à propos de son ouvrage La Laïcité scolaire (Berne, Peter LANG, 2008). J'avais proposé une analyse critique des thèses défendues dans ce livre, dans un article publié par la revue Penser l'éducation ( n$^{\circ}$ 25, Université de Rouen, mai 2009) sous le titre «École et Foulard. La laïcité est-elle une doctrine politique moderne ?». Philippe FORAY m'avait honoré d'une réponse à cette analyse dans le numéro suivant de la même revue ( $n^{\circ} 26$, décembre 2009) sous le titre "Laïcité et bien commun. Réponse à Roger Monjo ». Certes, il ne s'agit pas ici d'une "réponse à la réponse ", mais je me propose d'intégrer dans la présente contribution la discussion de certains éléments avancés par Philippe FORAY dans son article.

3. Voir, en particulier : « Religion et sphère publique », in Entre naturalisme et religion, GALLIMARD, 2008, article sur lequel nous nous appuierons plus particulièrement. Voir aussi : « Foi et savoir » in L'Avenir de la nature humaine, GALLIMARD, 2002; "De la tolérance religieuse aux droits

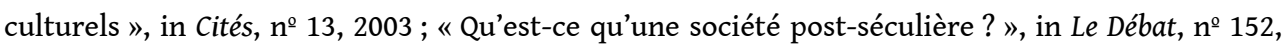
2008.

4. L'absence de prise en considération de cette dimension post-moderne (et post-séculière) dans la problématique actuelle de la laïcité me semble être le point le plus discutable dans l'argumentation de P. FORAY, tant dans son livre que dans sa réponse à mon analyse critique. Il est vrai que moi-même, dans celle-ci, je ne mets pas suffisamment ce point en avant. Aussi, je nuancerais aujourd'hui ma réponse à la question dont j'étais parti dans mon article : la laïcité estelle une doctrine politique moderne? Elle est bien une doctrine moderne, mais seulement au sens de la première modernité (qui est encore une modernité partielle, inachevée) et non au sens de la post-modernité (entendue comme pleine modernité).

5. Voir aussi, p. 13 : «Nous avons plus intérêt désormais à tenter de récupérer les contenus bibliques dans une foi de la raison qu'à combattre la soutane et l'obscurantisme ».

6. Jürgen HABERMAS évoque la portée tout à fait particulière, au lendemain de la rupture civilisationnelle qu'a constituée l'expérience nazie, du concept de "solidarité anamnétique » avec les victimes de torts passés, concept élaboré par W. BENJAMIN pour «combler le manque créé par l'espérance perdue en un jugement dernier » (idem, p. 52).

7. J'emprunte cette formule à J-M. FERRY qui, dans un article portant le titre « Sur le potentiel critique des religions dans l'espace européen » (in P. GISEL et J.-M. TETAZ (Ed.), Théories de la religion, Labor et Fides, Genève, 2002), défend un point de vue identique à celui de J. HABERMAS en écrivant par exemple : «[...] l'espace public doit ouvrir sans réserve les portes de la Cité aux intuitions jusqu'alors archivées dans le potentiel sémantique des religions ». Il précise un peu plus loin les raisons de cette nécessaire ouverture: "À l'heure où l'on sent venir le retour du refoulé, il devient 
paradoxalement urgent de mettre un terme à l'excommunication politique de la raison religieuse, si l'on veut en prévenir l'agitation ». Voir aussi, du même auteur, La religion réflexive, CERF, 2010.

8. Projet dont on sait, au demeurant, ce qu'il doit à certaines sources religieuses, en particulier la Réforme.

9. C'est un point que j'avais mis en exergue, dans mon article pour Penser l'éducation, de l'analyse que j'ai proposée des "affaires de foulard" à l'école en essayant de montrer, dans une perspective inspirée par la philosophie hégélienne, que le port du foulard pouvait être, initialement, interprété comme une demande de reconnaissance adressée à une instance qui était, par là même, préalablement reconnue comme légitime mais que cette demande avait essuyé une fin de non-recevoir à l'origine d'un possible sentiment d'injustice, dès lors que le débat qui s'en est suivi a davantage traité de la question de savoir quelle était la bonne réponse à apporter au problème créé par cette « interpellation » que de la bonne réponse à apporter à cette interpellation elle-même, entraînant de facto l'exclusion du débat des principaux-ales intéressé-es.

\section{RÉSUMÉS}

Cet article se propose, dans un premier temps, de mettre en lumière l'originalité de la notion de société post-séculière en posant, en particulier, la question de l'articulation de cette notion avec les théories traditionnelles de la sécularisation. Vise-t-elle à faire état d'un « retour du religieux»? Ou bien s'agit-il, au contraire, d'un approfondissement du processus de sécularisation lui-même? Dans un second temps, la question de l'application du principe de laïcité dans un tel contexte post-séculier est posée. Qu'en est-il de cette application dès lors que la laïcisation de la société, du moins dans le sens que ce projet a pris dans le cadre de l'idéologie française, n'apparaît plus comme le telos de la modernité?

To begin with, this article aims to highlight the concept of postsecular society by putting this concept into perspective with the traditional theories on secularism. Might we go as far as claiming that the postsecular society is characterised by a revival of religion? Or does it express on the contrary a deeper assertion of the process of secularism itself? Then, the article examines how the principle of secularism is implemented in the context of postsecularism. Indeed, how is this implementation achieved when the secularisation of society becomes the finality of modern times, at least in the specific sense this project acquires in the French ideology.

\section{INDEX}

Mots-clés : laïcité, religion, société post-séculière

Keywords : postsecular society, religion, secularism 
AUTEUR

ROGER MONJO

Maître de conférences

LIRDEF - CERFEE, Université Montpellier 3 